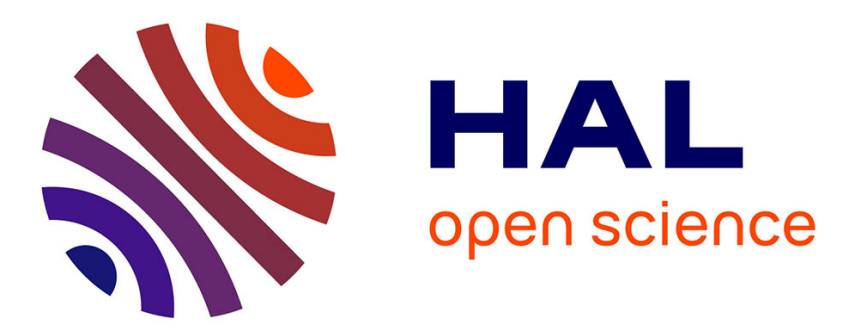

\title{
Reduced chaos decomposition with random coefficients of vector-valued random variables and random fields
}

\author{
Christian Soize, R. Ghanem
}

\section{To cite this version:}

Christian Soize, R. Ghanem. Reduced chaos decomposition with random coefficients of vector-valued random variables and random fields. Computer Methods in Applied Mechanics and Engineering, 2009, 98 (21-26), pp.1926-1934. 10.1016/j.cma.2008.12.035 . hal-00684487

\section{HAL Id: hal-00684487 \\ https://hal.science/hal-00684487}

Submitted on 2 Apr 2012

HAL is a multi-disciplinary open access archive for the deposit and dissemination of scientific research documents, whether they are published or not. The documents may come from teaching and research institutions in France or abroad, or from public or private research centers.
L'archive ouverte pluridisciplinaire HAL, est destinée au dépôt et à la diffusion de documents scientifiques de niveau recherche, publiés ou non, émanant des établissements d'enseignement et de recherche français ou étrangers, des laboratoires publics ou privés. 


\title{
Reduced Chaos Decomposition with Random Coefficients of Vector-Valued Random Variables and Random Fields
}

\author{
Christian Soize ${ }^{b}$ Roger G. Ghanem ${ }^{a, *}$ \\ a210 KAP Hall, University of Southern California, Los Angeles, CA 90089. \\ ${ }^{\mathrm{b}}$ Université Paris-Est, Laboratoire de Modélisation et Simulation Multi-Échelle, \\ MSME FRE3160 CNRS, 5 bd Descartes, Champs-sur-Marne, 77454 \\ Marne-la-Vallée, cedex 2
}

\begin{abstract}
We develop a stochastic functional representation that is adapted to problems involving various forms of epistemic uncertainties including modeling error and data paucity. The new representation builds on the polynomial chaos decomposition and eventually yields a Karhunen-Loeve expansion with random multiplicative coefficients. In this expansion, one set of uncertainty is captured in the usual manner, as uncorrelated scalar random variables. Another component of the uncertainty, statistically independent from the first, is captured by constructing the, usually deterministic, functions in the KL expansion as random functions. We think of the first set of uncertainties as associated with a coarse scale model, and of the second set as associated with subscale fluctuations not captured in the coarse scale description.
\end{abstract}

Key words: Uncertainty quantification; Polynomial Chaos; Karhunen-Loeve; Stochastic Model Reduction.

\section{Introduction}

A number of challenges remain on the path to achieving the impact of stochastic analysis for the treatment of complex systems whose behavior is subject to uncertainties. These challenges can be broadly classified into three groups:

\footnotetext{
* Corresponding author. Tel.: +2137409528.

Email addresses: christian.soize@univ-paris-est.fr (Christian Soize), ghanem@usc.edu (Roger G. Ghanem).
} 
Modeling, characterization, and propagation. In the modeling area, two further classifications can be identified. The first classification delineates between parametric and non-parametric interpretations of uncertainty. In the first case, uncertainty is attributed to stochastic fluctuations in the parameters of a physical model $[11,21]$. These fluctuations are typically interpreted as representing subscale fluctuations not fully resolved on the scale at which the governing physics is assumed to govern. In the second case, uncertainty is attributed to fluctuations in the form of the governing equations, leading to stochastic operator perturbations $[19,20,5]$. The second classification depends on the representative properties of the solution. Specifically, computed solutions can be representative of target solutions either in a distributional sense, a functional $\left(L^{2}\right)$ sense, or an almost-sure sense, with distinct sets of mathematical tools applicable depending on the case. More specifically, approximation accuracy, model reduction, and the ingredients of a well-posed problem should all be defined in the context of what is meant by equality (distributional, in-the-norm, or almost-sure) [1]. We note that stochastic $L^{2}$ representations provide a characterization of the solution of a given problem as a deterministic functional form in terms of the stochastic parameters. This form for the solution is significant if sensitivity information and $L^{2}$-style error analysis are subsequently required, but otherwise seems to provide too much information. Likewise, a distributional equivalence between the computed solution and the exact solution is suitable if the details of the stochastic degrees of freedom (i.e. stochastic dimensions) are not relevant to the final analysis. The most significant challenge associated with characterizing uncertainty consists in faithfully capturing the weight of available evidence, with error analysis capabilities to determine the value of additional evidence. Methods for characterizing uncertainty are typically adapted to specific modeling approaches and rely on methods of statistical analysis including statistical estimation and statistical inference. Procedures based on Maximum Likelihood [12,6,9], Bayesian inference [10], and Maximum Entropy [4,19] have been put in place to characterize mathematical models based on polynomial chaos decompositions and random matrix theory. The last challenge identified above relates to propagating uncertainty from data to predictions. This challenge has specific attributes depending on the interpretation of stochastic equality and on the specific stochastic model used. In all cases, however, it can be expected that the computational cost associated with this propagation step will grow with the complexity of the underlying stochastic data (requiring more stochastic degrees of freedom for a suitable characterization) and with the level of stochastic scatter in this data, typically resulting in a greater scatter in the predicted quantities of interest and a stronger nonlinear dependence of the predictions on the data.

Significant progress has been achieved in recent years in the analysis of stochastic partial differential equations with random coefficients. In particular, theory and algorithms underlying polynomial chaos and other functional approximation and projection methods have been set on firmer ground, providing a path forward towards a general purpose formulation of stochastic computational 
analysis $[8,23,14,13,3,15,7,2,22,16]$. Also, procedures for characterization and calibration of stochastic representations have been developed $[12,6,10,9,4]$ that are well-adapted to functional approximation methods and their significance to model validation has been demonstrated [9]. Most of these developments have been with a view to either calibrating probabilistic models or accelerating the convergence of uncertainty propagation procedures, including methodologies for a-priori and a-posteriori error estimation. Modeling efforts that address the curse of dimensionality for complex uncertainties and that provide a framework for integrating epistemic uncertainty into the same unified framework as other sources of uncertainty have only recently begun to emerge While a number of efforts have introduced polynomial chaos expansions where both the basis functions and their associated coordinates are random $[17,10,9]$. In these approaches, the mathematical analysis of these representations is still at a very fundamental level [18] that lags behind the nuances and sophistication of current practical needs. The present paper addresses this issue from a perspective that highlights its significance to problems of conceptual and mathematical modeling, calibration of probabilistic models, and computational efficiency. Specifically, we demonstrate how subset of the stochastic degrees of freedom can be rolled into the coefficients of a polynomial chaos expansion, thus permitting the segregation of the uncertainties for subsequent processing. For example, the uncertainties that are retained for functional approximation can be treated using stochastic Galerkin projections, while the uncertainties that have been rolled into the coefficients can be treated using distributional representation. This hybrid treatment of uncertainties will adapt the computational effort and algorithms to the specific needs of the problem at hand. The final byproduct of our analysis is a Karhunen-Loeve decomposition where the, usually deterministic, coordinate functions are now themselves stochastic, endowed with a probabilistic measure that is independent of the measure associated with the standard orthogonal Karhunen-Loeve random variables.

In a first part of the paper, we show how the reduced chaos decomposition with random coefficients of a $\mathbb{R}^{n}$-valued second-order random variable can be constructed and explore the mathematical properties of such a representation. A second part deals with the case of random fields. In the third part, we demonstrate our construction on an example that highlights some of its salient features. 


\section{Reduced chaos decomposition with random coefficients of a vector- valued second-order random variable}

\subsection{Chaos decomposition of a vector-valued second-order random variable on a tensor product of two Hilbert spaces}

In this subsection, we introduce the Chaos decomposition of a $\mathbb{R}^{n}$-valued second-order random variable with respect to the tensor product of two Hilbert spaces. This is a particular case of the more general setting analyzed in [21].

Let $\boldsymbol{X}=\left(X_{1}, \ldots, X_{n}\right), \boldsymbol{Y}=\left(Y_{1}, \ldots, Y_{m}\right)$ and $\boldsymbol{Z}=\left(Z_{1}, \ldots, Z_{p}\right)$ be three second-order random variables, defined on a probability space $(\Theta, \mathcal{T}, P)$, with values in $\mathbb{R}^{n}, \mathbb{R}^{m}$ and $\mathbb{R}^{p}$ respectively. The probability distributions on $\mathbb{R}^{n}$ and $\mathbb{R}^{m}$ of random variables $\boldsymbol{X}$ and $\boldsymbol{Y}$ are assumed to be given and are denoted by $P_{\boldsymbol{X}}(d \boldsymbol{x})$ and $P_{\boldsymbol{Y}}(d \boldsymbol{y})$, in which $d \boldsymbol{x}=d x_{1} \ldots d x_{n}$ and $d \boldsymbol{y}=d y_{1} \ldots d y_{m}$ are the Lebesgue measures on $\mathbb{R}^{n}$ and $\mathbb{R}^{m}$. It is assumed that random variables $\boldsymbol{X}$ and $\boldsymbol{Y}$ are independent. Consequently, the joint probability distribution on $\mathbb{R}^{n} \times \mathbb{R}^{m}$ of random variables $\boldsymbol{X}$ and $\boldsymbol{Y}$ is written as $P_{\boldsymbol{X}, \boldsymbol{Y}}(d \boldsymbol{x}, d \boldsymbol{y})=$ $P_{\boldsymbol{X}}(d \boldsymbol{x}) \otimes P_{\boldsymbol{Y}}(d \boldsymbol{y})$.

The random variable $\boldsymbol{Z}$ is assumed to be the transformation of $\boldsymbol{X}$ and $\boldsymbol{Y}$ by a measurable nonlinear mapping $(\boldsymbol{x}, \boldsymbol{y}) \mapsto \boldsymbol{f}(\boldsymbol{x}, \boldsymbol{y})=\left(f_{1}(\boldsymbol{x}, \boldsymbol{y}), \ldots, f_{p}(\boldsymbol{x}, \boldsymbol{y})\right)$ from $\mathbb{R}^{n} \times \mathbb{R}^{m}$ into $\mathbb{R}^{p}$, in which $\boldsymbol{x}=\left(x_{1}, \ldots, x_{n}\right)$ and $\boldsymbol{y}=\left(y_{1}, \ldots, y_{m}\right)$. We then have $\boldsymbol{Z}=\boldsymbol{f}(\boldsymbol{X}, \boldsymbol{Y})$.

Since $\boldsymbol{f}$ is such that $\boldsymbol{Z}$ is a second-order random variable, we have

$$
E\left\{\|\boldsymbol{f}(\boldsymbol{X}, \boldsymbol{Y})\|_{\mathbb{R}^{p}}^{2}\right\}=\int_{\mathbb{R}^{n}} \int_{\mathbb{R}^{m}}\|\boldsymbol{f}(\boldsymbol{x}, \boldsymbol{y})\|_{\mathbb{R}^{p}}^{2} P_{\boldsymbol{X}, \boldsymbol{Y}}(d \boldsymbol{x}, d \boldsymbol{y})<+\infty,
$$

in which $E\{$.$\} denotes the mathematical expectation, and where \|\cdot\|_{\mathbb{R}^{p}}$ denotes the Euclidean norm in $\mathbb{R}^{p}$ associated with the inner product $\left\langle\boldsymbol{z}, \boldsymbol{z}^{\prime}\right\rangle_{\mathbb{R}^{p}}=z_{1} z_{1}^{\prime}+$ $\ldots+z_{p} z_{p}^{\prime}$. From Eq. (1), it can be deduced that mapping $\boldsymbol{f}$ belongs to the space $L_{P_{\boldsymbol{X}, \boldsymbol{Y}}}^{2}\left(\mathbb{R}^{n} \times \mathbb{R}^{m}, \mathbb{R}^{p}\right)$ of $P_{\boldsymbol{X}, \boldsymbol{Y}^{-}}$square-integrable functions from vector space $\mathbb{R}^{n} \times \mathbb{R}^{m}$ into vector space $\mathbb{R}^{p}$.

Let $\mathbb{H}_{X}=L_{P_{X}}^{2}\left(\mathbb{R}^{n}\right)$ (resp. $\mathbb{H}_{Y}=L_{P_{Y}}^{2}\left(\mathbb{R}^{m}\right)$ ) be the real Hilbert space of $P_{\boldsymbol{X}^{-}}$ square-integrable functions (resp. $P_{Y}$-square-integrable functions) from vector space $\mathbb{R}^{n}$ (resp. $\mathbb{R}^{m}$ ) into $\mathbb{R}$. The real Hilbert spaces $\mathbb{H}_{X}$ and $\mathbb{H}_{Y}$ are equipped with the following inner products:

$$
\begin{aligned}
\left\langle u, u^{\prime}\right\rangle_{\mathbb{H}_{X}} & =\int_{\mathbb{R}^{n}} u(\boldsymbol{x}) u^{\prime}(\boldsymbol{x}) P_{\boldsymbol{X}}(d \boldsymbol{x}) \\
& =E\left\{u(\boldsymbol{X}) u^{\prime}(\boldsymbol{X})\right\}
\end{aligned}
$$




$$
\begin{aligned}
\left\langle v, v^{\prime}\right\rangle_{\mathbb{H}_{Y}} & =\int_{\mathbb{R}^{m}} v(\boldsymbol{y}) v^{\prime}(\boldsymbol{y}) P_{\boldsymbol{Y}}(d \boldsymbol{y}) \\
& =E\left\{v(\boldsymbol{Y}) v^{\prime}(\boldsymbol{Y})\right\}
\end{aligned}
$$

We introduce the multi-index $\boldsymbol{\alpha}=\left(\alpha_{1}, \ldots, \alpha_{n}\right) \in \mathbb{N}^{n}$ and the multi-index $\boldsymbol{\beta}=\left(\beta_{1}, \ldots, \beta_{m}\right) \in \mathbb{N}^{m}$. Let us consider a Hilbertian basis of real Hilbert space $\mathbb{H}_{X}\left(\right.$ resp. $\left.\mathbb{H}_{Y}\right)$ given by $\left\{\varphi_{\boldsymbol{\alpha}}, \boldsymbol{\alpha} \in \mathbb{N}^{n}\right\}$ (resp. $\left\{\psi_{\boldsymbol{\beta}}, \boldsymbol{\beta} \in \mathbb{N}^{m}\right\}$ ). We thus have

$$
\begin{gathered}
\left\langle\varphi_{\boldsymbol{\alpha}}, \varphi_{\boldsymbol{\alpha}^{\prime}}\right\rangle_{\mathbb{H}_{X}}=E\left\{\varphi_{\boldsymbol{\alpha}}(\boldsymbol{X}) \varphi_{\boldsymbol{\alpha}^{\prime}}(\boldsymbol{X})\right\}=\delta_{\boldsymbol{\alpha} \boldsymbol{\alpha}^{\prime}}, \\
\left\langle\psi_{\boldsymbol{\beta}}, \psi_{\boldsymbol{\beta}^{\prime}}\right\rangle_{\mathbb{H}_{Y}}=E\left\{\psi_{\boldsymbol{\beta}}(\boldsymbol{Y}) \psi_{\boldsymbol{\beta}^{\prime}}(\boldsymbol{Y})\right\}=\delta_{\boldsymbol{\beta} \boldsymbol{\beta}^{\prime}} .
\end{gathered}
$$

Therefore any function $g \in \mathbb{H}_{X}$ (resp. $h \in \mathbb{H}_{Y}$ ) can be expanded as

$$
g(\boldsymbol{x})=\sum_{\boldsymbol{\alpha} \in \mathbb{N}^{n}} g_{\boldsymbol{\alpha}} \varphi_{\boldsymbol{\alpha}}(\boldsymbol{x}) \quad, \quad h(\boldsymbol{y})=\sum_{\boldsymbol{\beta} \in \mathbb{N}^{m}} h_{\boldsymbol{\beta}} \psi_{\boldsymbol{\beta}}(\boldsymbol{y})
$$

in which

$$
g_{\boldsymbol{\alpha}}=\left\langle g, \varphi_{\boldsymbol{\alpha}}\right\rangle_{\mathbb{H}_{X}}=E\left\{g(\boldsymbol{X}) \varphi_{\boldsymbol{\alpha}}(\boldsymbol{X})\right\}, \quad h_{\boldsymbol{\beta}}=\left\langle h, \psi_{\boldsymbol{\beta}}\right\rangle_{\mathbb{H}_{Y}}=E\left\{h(\boldsymbol{Y}) \psi_{\boldsymbol{\beta}}(\boldsymbol{Y})\right\}
$$

Below, it is assumed that the Hilbertian bases $\varphi_{\boldsymbol{\alpha}}$ and $\psi_{\boldsymbol{\beta}}$ are polynomial bases such that

$$
\varphi_{\mathbf{0}}(\boldsymbol{X})=1 \quad, \quad \psi_{\mathbf{0}}(\boldsymbol{Y})=1
$$

Consequently, we deduce that

$$
E\left\{\varphi_{\boldsymbol{\alpha}}(\boldsymbol{X})\right\}=0, \forall \boldsymbol{\alpha} \neq \mathbf{0} \text { and } E\left\{\psi_{\boldsymbol{\beta}}(\boldsymbol{Y})\right\}=0, \forall \boldsymbol{\beta} \neq \mathbf{0}
$$

It can then be proven [21] that the $\mathbb{R}^{p}$-valued random variable $\boldsymbol{Z}=\boldsymbol{f}(\boldsymbol{X}, \boldsymbol{Y})$ has the following Chaos representation related to the tensor product of $\mathbb{H}_{X}$ with $\mathbb{H}_{Y}$,

$$
\boldsymbol{Z}=\sum_{\boldsymbol{\alpha} \in \mathbb{N}^{n}} \sum_{\boldsymbol{\beta} \in \mathbb{N}^{m}} z^{\boldsymbol{\alpha} \boldsymbol{\beta}} \varphi_{\boldsymbol{\alpha}}(\boldsymbol{X}) \psi_{\boldsymbol{\beta}}(\boldsymbol{Y})
$$

in which the coefficients $\boldsymbol{z}^{\boldsymbol{\alpha} \boldsymbol{\beta}} \in \mathbb{R}^{p}$ are given by

$$
\boldsymbol{z}^{\boldsymbol{\alpha} \boldsymbol{\beta}}=E\left\{\boldsymbol{Z} \varphi_{\boldsymbol{\alpha}}(\boldsymbol{X}) \psi_{\boldsymbol{\beta}}(\boldsymbol{Y})\right\}
$$

We then have the following properties concerning the second-order moments of random variable $\boldsymbol{Z}$,

$$
E\{\boldsymbol{Z}\}=\boldsymbol{z}_{\mathbf{0 0}} \quad, \quad E\left\{\boldsymbol{Z} \boldsymbol{Z}^{T}\right\}=\sum_{\boldsymbol{\alpha} \in \mathbb{N}^{n}} \sum_{\boldsymbol{\beta} \in \mathbb{N}^{m}} \boldsymbol{z}^{\boldsymbol{\alpha} \boldsymbol{\beta}}\left(\boldsymbol{z}^{\boldsymbol{\alpha} \boldsymbol{\beta}}\right)^{T}
$$

which yields

$$
E\left\{\|\boldsymbol{Z}\|_{\mathbb{R}^{p}}^{2}\right\}=\sum_{\boldsymbol{\alpha} \in \mathbb{N}^{n}} \sum_{\boldsymbol{\beta} \in \mathbb{N}^{m}}\left\|\boldsymbol{z}^{\boldsymbol{\alpha} \boldsymbol{\beta}}\right\|_{\mathbb{R}^{p}}^{2}<+\infty
$$


2.2 Existence and properties of the Chaos decomposition with random coefficients of a vector-valued second-order random variable

We have the following proposition related to the existence and the properties of the Chaos decomposition with random coefficients of a vector-valued secondorder random variable.

Proposition 1 Let $\boldsymbol{Z}=\left(Z_{1}, \ldots, Z_{p}\right)$ be the $\mathbb{R}^{p}$-valued second-order random variable defined in Subsection 2.1. Then there exists a chaos decomposition with random coefficients of random variable $\boldsymbol{Z}$ which is written as

$$
Z=\sum_{\boldsymbol{\alpha} \in \mathbb{N}^{n}} A^{\boldsymbol{\alpha}} \varphi_{\boldsymbol{\alpha}}(\boldsymbol{X})
$$

in which $\left\{\boldsymbol{A}^{\boldsymbol{\alpha}}, \boldsymbol{\alpha} \in \mathbb{N}^{n}\right\}$ (1) is a sequence of dependent $\mathbb{R}^{p}$-valued random variables, (2) is independent of the $\mathbb{R}^{n}$-valued random variable $\boldsymbol{X}$ and (3) is such that

$$
E\left\{\boldsymbol{A}^{\mathbf{0}}\right\}=E\{\boldsymbol{Z}\} \quad, \quad E\left\{\sum_{\boldsymbol{\alpha} \in \mathbb{N}^{n}}\left\|\boldsymbol{A}^{\boldsymbol{\alpha}}\right\|_{\mathbb{R}^{p}}^{2}\right\}=E\left\{\|\boldsymbol{Z}\|_{\mathbb{R}^{p}}^{2}\right\}
$$

Proof. Introducing the sequence $\left\{\boldsymbol{A}^{\boldsymbol{\alpha}}, \boldsymbol{\alpha} \in \mathbb{N}^{n}\right\}$ of the $\mathbb{R}^{p}$-valued random variables such that

$$
\boldsymbol{A}^{\boldsymbol{\alpha}}=\sum_{\boldsymbol{\beta} \in \mathbb{N}^{m}} \boldsymbol{z}^{\boldsymbol{\alpha} \boldsymbol{\beta}} \psi_{\boldsymbol{\beta}}(\boldsymbol{Y})
$$

and taking into account Eq. (10) yield Eq. (14). Clearly, all the random variables $\left\{\boldsymbol{A}^{\boldsymbol{\alpha}}, \boldsymbol{\alpha} \in \mathbb{N}^{n}\right\}$ are independent of random variable $\boldsymbol{X}$ because the random variables $\boldsymbol{X}$ and $\boldsymbol{Y}$ are independent. Since random variables $\left\{\boldsymbol{A}^{\boldsymbol{\alpha}}, \boldsymbol{\alpha} \in\right.$ $\left.\mathbb{N}^{n}\right\}$ are independent of $\boldsymbol{X}$, we have $E\{\boldsymbol{Z}\}=\sum_{\boldsymbol{\alpha} \in \mathbb{N}^{n}} E\left\{\boldsymbol{A}^{\boldsymbol{\alpha}}\right\} E\left\{\varphi_{\boldsymbol{\alpha}}(\boldsymbol{X})\right\}$ and then using Eqs. (8) and (9) yield the first part of Eq. (15). From Eq. (14) we have $E\left\{\|\boldsymbol{Z}\|_{\mathbb{R}^{p}}^{2}\right\}=\sum_{\boldsymbol{\alpha} \in \mathbb{N}^{n}} \sum_{\boldsymbol{\alpha}^{\prime} \in \mathbb{N}^{n}} E\left\{\left\langle\boldsymbol{A}^{\boldsymbol{\alpha}}, \boldsymbol{A}^{\boldsymbol{\alpha}^{\prime}}\right\rangle_{\mathbb{R}^{p}}\right\} E\left\{\varphi_{\boldsymbol{\alpha}}(\boldsymbol{X}) \varphi_{\boldsymbol{\alpha}^{\prime}}(\boldsymbol{X})\right\}$. Using Eq. (4) yields the second part of Eq. (15).

2.3 Reduced chaos decomposition with random coefficients of a vector-valued second-order random variable

We introduce the finite-dimensional approximation $\boldsymbol{Z}^{\nu}$ of order $\nu$ of random variable $\boldsymbol{Z}$ whose chaos decomposition with random coefficients is given by Eq. (14). This approximation is then defined by

$$
Z^{\nu}=\sum_{\boldsymbol{\alpha} \in \mathbb{N}^{n},|\alpha| \leq \nu} A^{\alpha} \varphi_{\boldsymbol{\alpha}}(\boldsymbol{X})
$$


in which $|\boldsymbol{\alpha}| \leq \nu$ means $0 \leq \alpha_{1}+\ldots+\alpha_{n} \leq \nu$. Below, the set of multi-indexes $|\boldsymbol{\alpha}| \leq \nu$ will mean the finite subset of $\mathbb{N}^{n}$ such that $|\boldsymbol{\alpha}| \leq \nu$.

Let $\boldsymbol{B}$ be the random vector made up of the random vectors $\left(\boldsymbol{A}^{\boldsymbol{\alpha}},|\boldsymbol{\alpha}| \leq \nu\right)$. Let $\boldsymbol{m}_{\boldsymbol{B}}=E\{\boldsymbol{B}\}$ be the mean value and let $\left[C_{\boldsymbol{B}}\right]=E\left\{\left(\boldsymbol{B}-\boldsymbol{m}_{\boldsymbol{B}}\right)\left(\boldsymbol{B}-\boldsymbol{m}_{\boldsymbol{B}}\right)^{T}\right\}$ be the covariance matrix of random vector $\boldsymbol{B}$. Assuming that none of the vectors $\boldsymbol{A}^{\boldsymbol{\alpha}}$ are deterministic, the symmetric covariance matrix $\left[C_{\boldsymbol{B}}\right]$ is positive definite. Under this assumption, we introduce the eigenvalue problem related to the positive definite symmetric covariance matrix,

$$
\left[C_{\boldsymbol{B}}\right] \boldsymbol{b}^{j}=\lambda_{j} \boldsymbol{b}^{j} \quad, \quad \lambda_{1} \geq \lambda_{2} \geq \ldots \geq \lambda_{N} \geq \ldots,
$$

in which the associated eigenvectors $\boldsymbol{b}^{1}, \boldsymbol{b}^{2}, \ldots, \boldsymbol{b}^{N}, \ldots$ form an orthonormal basis. Introducing the bloc decomposition $\boldsymbol{b}^{j}=\left(\boldsymbol{a}^{\boldsymbol{\alpha}, j},|\boldsymbol{\alpha}| \leq \nu\right)$, we then have

$$
\left\langle\boldsymbol{b}^{j}, \boldsymbol{b}^{j^{\prime}}\right\rangle=\sum_{\boldsymbol{\alpha},|\boldsymbol{\alpha}| \leq \nu}\left\langle\boldsymbol{a}^{\boldsymbol{\alpha}, j}, \boldsymbol{a}^{\boldsymbol{\alpha}, j^{\prime}}\right\rangle_{\mathbb{R}^{p}}=\delta_{j j^{\prime}}
$$

Finally, the following approximation $\boldsymbol{B}^{N}$ of order $N \ll \nu$ for random vector $\boldsymbol{B}$ is introduced,

$$
\boldsymbol{B}^{N}=\boldsymbol{m}_{\boldsymbol{B}}+\sum_{j=1}^{N} \sqrt{\lambda_{j}} \xi_{j} \boldsymbol{b}^{j}
$$

in which the random variables $\xi_{1}, \ldots, \xi_{N}$ satisfy the following usual properties,

$$
E\left\{\xi_{j}\right\}=0 \quad, \quad E\left\{\xi_{j} \xi_{j^{\prime}}\right\}=\delta_{j j^{\prime}} .
$$

Using the bloc decomposition, Eq. (20) yields for all $\boldsymbol{\alpha}$ in $\mathbb{N}^{n}$ such that $|\boldsymbol{\alpha}| \leq \nu$,

$$
\boldsymbol{A}^{\boldsymbol{\alpha}, N}=E\left\{\boldsymbol{A}^{\boldsymbol{\alpha}}\right\}+\sum_{j=1}^{N} \sqrt{\lambda_{j}} \xi_{j} \boldsymbol{a}^{\boldsymbol{\alpha}, j}
$$

The corresponding approximation $\boldsymbol{Z}^{\nu, N}$ of random vector $\boldsymbol{Z}^{\nu}$ defined by Eq. (17) is then written as

$$
\boldsymbol{Z}^{\nu, N}=\sum_{\boldsymbol{\alpha},|\boldsymbol{\alpha}| \leq \nu} \boldsymbol{A}^{\boldsymbol{\alpha}, N} \varphi_{\boldsymbol{\alpha}}(\boldsymbol{X})
$$

Proposition 2 The reduced chaos decomposition with random coefficients of order $\{\nu, N\}$ for the $\mathbb{R}^{p}$-valued second-order random variable $\boldsymbol{Z}$ defined in Subsection 2.1 is written as

$$
\boldsymbol{Z}^{\nu, N}=\boldsymbol{\Phi}^{0}(\boldsymbol{X})+\sum_{j=1}^{N} \sqrt{\lambda_{j}} \xi_{j} \boldsymbol{\Phi}^{j}(\boldsymbol{X})
$$

in which $\xi_{1}, \ldots, \xi_{N}$ are second-order, centered and uncorrelated real-valued random variables satisfying Eq. (21) and where $\boldsymbol{\Phi}^{0}(\boldsymbol{X}), \boldsymbol{\Phi}^{1}(\boldsymbol{X}), \ldots, \boldsymbol{\Phi}^{N}(\boldsymbol{X})$ are $N+1$ random vectors with values in $\mathbb{R}^{p}$ such that for all $j$ and $j^{\prime}$ in $\{1, \ldots, N\}$,

$$
E\left\{\left\langle\Phi^{j}(\boldsymbol{X}), \Phi^{j^{\prime}}(\boldsymbol{X})\right\rangle_{\mathbb{R}^{p}}\right\}=\delta_{j j^{\prime}}
$$


Random vectors $\boldsymbol{\Phi}^{0}(\boldsymbol{X}), \boldsymbol{\Phi}^{1}(\boldsymbol{X}), \ldots, \boldsymbol{\Phi}^{N}(\boldsymbol{X})$ are such that

$\boldsymbol{\Phi}^{0}(\boldsymbol{X})=\sum_{\boldsymbol{\alpha},|\boldsymbol{\alpha}| \leq \nu} E\left\{\boldsymbol{A}^{\boldsymbol{\alpha}}\right\} \varphi_{\boldsymbol{\alpha}}(\boldsymbol{X}) \quad, \quad \boldsymbol{\Phi}^{j}(\boldsymbol{X})=\sum_{\boldsymbol{\alpha},|\boldsymbol{\alpha}| \leq \nu} \boldsymbol{a}^{\boldsymbol{\alpha}, j} \varphi_{\boldsymbol{\alpha}}(\boldsymbol{X}) \quad, \quad 1 \leq j \leq N$.

Proof. Substituting Eq. (22) into Eq. (23) yields Eq. (24) with Eq. (26). From Eqs. (4), (19) and (26), it can be deduced Eq. (25).

\section{Reduced chaos decomposition with random coefficients of ran- dom fields}

Consider next the stochastic process $u(x(t), y(t)), t \in \mathcal{T} \subset \mathbb{R}$. We will assume that $u, x(t)$ and $y(t)$ are all second-order processes indexed by $\mathcal{T}$, signifying that each of their covariance operators is Hilbert-Schmidt (or of trace type). We will also assume that stochastic processes $x(t)$ and $y(t)$ are statistically independent. Under this assumption, for all $t$ in $\mathcal{T}, x(t)$ and $y(t)$ can be approximated by their respective truncated Karhunen-Loeve expansions as follows,

$$
x(t)=m_{x}(t)+\sum_{i=1}^{n} X_{i} \sqrt{\rho}_{i} e_{i}(t)
$$

and

$$
y(t)=m_{y}(t)+\sum_{i=1}^{m} Y_{i} \sqrt{\gamma}_{i} f_{i}(t)
$$

where $\left(\rho_{i}, e_{i}\right)$ and $\left(\gamma_{i}, f_{i}\right)$ are, respectively, the eigenvalue-eigenvector pairs of the covariance operators of $x(t)$ and $y(t)$ and where $m_{x}(t)$ and $m_{y}(t)$ are their respective mean functions. We will rewrite $u(x(t), y(t))$ as $u_{n, m}(\boldsymbol{X}, \boldsymbol{Y}, t)$ in order to highlight this decomposition, where $\boldsymbol{X}=\left(X_{1}, \cdots, X_{n}\right)$ and $\boldsymbol{Y}=$ $\left(Y_{1}, \cdots, Y_{m}\right)$ are, respectively, random variables with values in $\mathbb{R}^{n}$ - and $\mathbb{R}^{m}$. The polynomial chaos decomposition of the second-order process $u_{n, m}$ can be written as

$$
u_{n, m}(\boldsymbol{X}, \boldsymbol{Y}, t)=\sum_{\boldsymbol{\alpha} \in \mathbb{N}^{n}} \sum_{\boldsymbol{\beta} \in \mathbb{N}^{m}} u_{n, m}^{\boldsymbol{\alpha} \boldsymbol{\beta}}(t) \varphi_{\boldsymbol{\alpha}}(\boldsymbol{X}) \psi_{\boldsymbol{\beta}}(\boldsymbol{Y}) .
$$

With the obvious notation, this equation can be rewritten as,

$$
u_{n, m}(\boldsymbol{X}, \boldsymbol{Y}, t)=\sum_{\boldsymbol{\alpha} \in \mathbb{N}^{n}} A_{n, m}^{\boldsymbol{\alpha}}(t) \varphi_{\boldsymbol{\alpha}}(\boldsymbol{X}),
$$


where random processes $A_{n, m}^{\alpha}(t)$ depend on random variables $\boldsymbol{Y}$ and are independent of $\boldsymbol{X}$. It is clear how the development from the previous section can then be extended to the analysis of the stochastic processes considered in this section. We note that in the present case, $A_{n, m}^{\boldsymbol{\alpha}}(t)$ are second-order random processes, and not random variables, as was the case previously. Truncating the summation in equation (30) to polynomials of at most order $\nu$ yields the $\nu^{\text {th }}$-order approximation,

$$
u_{n, m}^{\nu}(\boldsymbol{X}, \boldsymbol{Y}, t)=\sum_{\boldsymbol{\alpha} \in \mathbb{N}^{n},|\boldsymbol{\alpha}| \leq \nu} A_{n, m}^{\boldsymbol{\alpha}}(t) \varphi_{\boldsymbol{\alpha}}(\boldsymbol{X}) .
$$

We note again that the random processes $A_{n, m}^{\alpha}(t)$ are correlated, and we seek to reduce them through a Karhunen-Loeve decomposition. We introduce the multivariate random process $\boldsymbol{B}(t)$ composed of the random processes $\left(A_{n, m}^{\boldsymbol{\alpha}}(t),|\boldsymbol{\alpha}| \leq \nu\right)$, and consider its covariance $\left[\boldsymbol{C}_{\boldsymbol{B}}(t, s)\right]=E\{(\boldsymbol{B}(t)-$ $\left.\left.\boldsymbol{m}_{\boldsymbol{B}}(t)\right)\left(\boldsymbol{B}(s)-\boldsymbol{m}_{\boldsymbol{B}}(s)\right)^{T}\right\}$, where $\boldsymbol{m}_{\boldsymbol{B}}(t)$ denotes the mean of random process $\boldsymbol{B}(t)$. Denoting by integers $p$ and $q$ the respective indices of the multi-indices $\boldsymbol{p}$ and $\boldsymbol{q}$, the $(p, q)$ entry of $\boldsymbol{C}_{\boldsymbol{B}}(t, s)$ is given by

$$
\begin{aligned}
{\left[\boldsymbol{C}_{\boldsymbol{B}}(t, s)\right]_{p, q} } & =E\left\{A_{n, m}^{\boldsymbol{p}}(t) A_{n, m}^{\boldsymbol{q}}(s)\right\} \\
& =\sum_{\boldsymbol{\alpha} \in \mathbb{N}^{n},|\boldsymbol{\alpha}| \leq \nu} u_{n, m}^{\boldsymbol{\alpha p}}(t) u_{n, m}^{\boldsymbol{\alpha q}}(s)
\end{aligned}
$$

The eigenproblem of the covariance operator of $\boldsymbol{B}(t)$ can then be written as,

$$
\int_{\mathcal{T}}\left[\boldsymbol{C}_{\boldsymbol{B}}(t, s)\right] \boldsymbol{b}^{j}(s) d s=\lambda_{j} \boldsymbol{b}^{j}(t)
$$

Random process $\boldsymbol{B}(t)$ can then be written as

$$
\boldsymbol{B}(t)=\boldsymbol{m}_{\boldsymbol{B}}(t)+\sum_{j=1}^{N} \sqrt{\lambda_{j}} \xi_{j} \boldsymbol{b}^{j}(t) .
$$

Denoting the $\boldsymbol{\alpha}$-block of $\boldsymbol{b}^{j}(t)$ by $a^{\boldsymbol{\alpha}, j}(t)$, a corresponding approximation of $A_{n, m}^{\alpha}(t)$ is obtained in the form,

$$
A_{n, m}^{\boldsymbol{\alpha}, N}(t)=E\left\{A^{\boldsymbol{\alpha}}(t)\right\}+\sum_{j=1}^{N} \sqrt{\lambda_{j}} \xi_{j} a^{\boldsymbol{\alpha}, j}(t) .
$$

Clearly, in this last equation, the random variables $\left\{\xi_{i}\right\}$ are functions of the random variables $\boldsymbol{Y}$. Substituting into equation (31), yields the following proposition.

Proposition 3 The reduced chaos decomposition with random coefficients of order $\{\nu, N\}$ for the second-order stochastic process $u(t)$ defined in Section 3 
is written as

$$
u_{n, m}^{\nu, N}(\boldsymbol{X}, \boldsymbol{Y}, t)=\Phi^{0}(\boldsymbol{X}, t)+\sum_{j=1}^{N} \sqrt{\lambda_{j}} \xi_{j} \Phi^{j}(\boldsymbol{X}, t)
$$

in which $\xi_{1}, \ldots, \xi_{N}$ are second-order, centered and uncorrelated real-valued random variables satisfying $E q$. (21) and where $\Phi^{0}(\boldsymbol{X}, t), \Phi^{1}(\boldsymbol{X}, t), \ldots, \Phi^{N}(\boldsymbol{X}, t)$ are $N+1$ second-order random processes such that for all $j$ and $j^{\prime}$ in $\{1, \ldots, N\}$,

$$
E\left\{\int_{\mathcal{T}} \Phi^{j}(\boldsymbol{X}, t) \Phi^{j^{\prime}}(\boldsymbol{X}, t) d t\right\}=\delta_{j j^{\prime}}
$$

Random processes $\Phi^{0}(\boldsymbol{X}, t), \Phi^{1}(\boldsymbol{X}, t), \ldots, \Phi^{N}(\boldsymbol{X}, t)$ are such that

$\Phi^{0}(\boldsymbol{X}, t)=\sum_{\boldsymbol{\alpha},|\boldsymbol{\alpha}| \leq \nu} E\left\{A^{\boldsymbol{\alpha}}(t)\right\} \varphi_{\boldsymbol{\alpha}}(\boldsymbol{X}) \quad, \quad \Phi^{j}(\boldsymbol{X}, t)=\sum_{\boldsymbol{\alpha},|\boldsymbol{\alpha}| \leq \nu} a^{\boldsymbol{\alpha}, j}(t) \varphi_{\boldsymbol{\alpha}}(\boldsymbol{X}) \quad, \quad 1 \leq j \leq N \quad$.

\section{Numerical Example}

Consider the random process,

$$
u(\boldsymbol{\xi}, \boldsymbol{\eta}, t)=\int_{0}^{t} \frac{\sum_{i \in \mathcal{C}_{1}} \xi_{i} e_{i}(s)}{\sum_{j \in \mathcal{C}_{2}} \eta_{j} f_{j}(s)} d s
$$

where $\mathcal{C}_{1}$ and $\mathcal{C}_{2}$ are two subsets of $\mathbb{N}$ of cardinality $n$ and $m$, respectively, and $\left\{e_{i}(t)\right\}$ and $\left\{f_{j}(t)\right\}$ are deterministic functions. Furthermore, $\boldsymbol{\xi}=\left(\xi_{i}\right)_{i \in \mathcal{C}_{1}}$ and $\boldsymbol{\eta}=\left(\eta_{j}\right)_{j \in \mathcal{C}_{2}}$ are, respectively, second-order $\mathbb{R}^{n}$ and $\mathbb{R}^{m}$-valued random variables, with $\boldsymbol{\xi}$ and $\boldsymbol{\eta}$ statistically independent. With this expression, $u(\boldsymbol{\xi}, \boldsymbol{\eta}, t)$ could represent, for example, the deflection of a one-dimensional bar subjected to a random body force given by $\sum_{i \in \mathcal{C}_{1}} \xi_{i} e_{i}(t)$ and with a random section modulus given by $\sum_{j \in \mathcal{C}_{2}} \eta_{j} f_{j}(t)$. The deterministic functions $\left\{e_{i}(t)\right\}$ and $\left\{f_{j}(t)\right\}$ can be thought of as characterizing length scales associated with the corresponding random variables. These can be due, for instance, to variabilities in specific dominant subscale features. We specifically assume that the random variables $\left\{\xi_{i}\right\}$ and $\left\{\eta_{j}\right\}$ are each distributed with an independent uniform distribution over $[0,1]$. Next, we consider the polynomial chaos decomposition of $u(\boldsymbol{\xi}, \boldsymbol{\eta}, t)$ in the form,

$$
u(\boldsymbol{\xi}, \boldsymbol{\eta}, t)=\sum_{\boldsymbol{\alpha} \in \mathbb{N}^{n}} \sum_{\boldsymbol{\beta} \in \mathbb{N}^{m}} u^{\boldsymbol{\alpha} \boldsymbol{\beta}}(t) \varphi_{\boldsymbol{\alpha}}(\boldsymbol{\xi}) \psi_{\boldsymbol{\beta}}(\boldsymbol{\eta})
$$

where $\varphi_{\boldsymbol{\alpha}}$ and $\psi_{\boldsymbol{\beta}}$ are the modified Legendre polynomials, orthonormal with respect to the multi-dimensional Lebesgue measure over the hypercube $[0,1]$. 


\begin{tabular}{ccc}
\hline $\mathrm{i}$ & $e_{i}(t)$ & $f_{i}(t)$ \\
0 & 1 & 1 \\
1 & $0.1 \cos (\pi t)$ & $0.2 \cos (\pi t)$ \\
2 & $0.002 \cos (10 \pi t)$ & $0.1 \cos (10 \pi t)$ \\
3 & $0.001 \cos (50 \pi t)$ & $0.01 \cos (20 \pi t)$ \\
4 & $0.0001 \cos (100 \pi t)$ & $0.002 \cos (30 \pi t)$ \\
5 & & $0.001 \cos (40 \pi t)$ \\
6 & $0.0002 \cos (50 \pi t)$ \\
7 & $0.0002 \cos (60 \pi t)$ \\
8 & $0.0002 \cos (70 \pi t)$ \\
9 & & $0.0002 \cos (80 \pi t)$ \\
10 & & $0.0002 \cos (90 \pi t)$ \\
11 & & $0.0002 \cos (100 \pi t)$ \\
12 & & $0.0002 \cos (110 \pi t)$ \\
13 & & $0.0002 \cos (120 \pi t)$ \\
14 & & $0.0002 \cos (130 \pi t)$ \\
15 & $0.0002 \cos (140 \pi t)$ \\
16 & & $0.0002 \cos (150 \pi t)$ \\
10 & &
\end{tabular}

Table 1

Data used in constructing the numerical example.

These can be obtained as tensor products from their one-dimensional counterparts. In view of this orthonormality, the coefficients $u^{\alpha \beta}$ can be evaluated as

$$
u^{\alpha \boldsymbol{\beta}}(t)=E\left\{u(\boldsymbol{\xi}, \boldsymbol{\eta}, t) \varphi_{\boldsymbol{\alpha}}(\boldsymbol{\xi}) \psi_{\boldsymbol{\beta}}(\boldsymbol{\eta})\right\} .
$$

The right hand side in this last expression cannot, in general, be evaluated analytically. In the present example, we evaluate the expectation operator through statistical sampling, taking an average over $10^{6}$ samples. Furthermore, in the present case, we consider the case where $n=4, m=16$, and a complete 20-dimensional second order polynomial in $\boldsymbol{\xi}$ and $\boldsymbol{\eta}$ is considered for the representation of $u(\boldsymbol{\xi}, \boldsymbol{\eta}, t)$. The data used in the present example are shown in Table 1. Given the orthogonality of both the deterministic functions and the random variables appearing in the integrand in equation (39), the numerator and the denominator inside the integral sign in that expression can be viewed as providing a Karhunen-Loeve representation of some underlying stochastic process. 
Next we consider the construction of a reduced-order model of the stochastic process $u$ that is explicitly dependent on a subset of the $\boldsymbol{\xi}$ and $\boldsymbol{\eta}$ variables, with the other variables being implicitly represented through the statistical dependence of the coefficients. Let $\boldsymbol{X}=\left(\xi_{1}, \eta_{1}, \eta_{2}, \eta_{3}\right)$ and let $\boldsymbol{Y}$ be a vector of random variables consisting of all the other elements of the $\boldsymbol{\xi}$ and $\boldsymbol{\eta}$ vectors. We have thus placed in $\boldsymbol{X}$ those random variables whose contribution is expected to be significant on $u(\boldsymbol{\xi}, \boldsymbol{\eta}, t)$ and we retained the other variables into vector $\boldsymbol{Y}$. With some abuse of notation, and for consistency with the notation of the previous sections, we refer to the process $u$ as $u(\boldsymbol{X}, \boldsymbol{Y}, t)$. In addition to this layout, we also consider a case where $\boldsymbol{X}=\left(\xi_{2}, \eta_{2}, \eta_{3}, \eta_{4}\right)$ and another case where $\boldsymbol{X}=\left(\xi_{4}, \eta_{8}, \eta_{9}, \eta_{10}\right)$. We will refer to these three cases as cases 1, 2 and 3, respectively. Case 1 corresponds to the situation where the coarse scale variables are the dominant variables representing the physical problem. Cases 2 and 3 refer, respectively, to situations where increasingly less important variables are identified as coarse scale observables. For each of these three cases, a four-dimensional second-order polynomial representation is constructed, as indicated above, whose coefficients are random variables, dependent on the remaining 16 variables. For each of these cases. Such a representation takes the following explicit form,

$$
\begin{aligned}
u(\boldsymbol{X}, \boldsymbol{Y}, t) & =\left(u^{00}(t)+\sum_{\alpha=1}^{16} u^{1 \alpha}(t) P_{1}\left(Y_{\alpha}\right)+\sum_{\alpha=1}^{16} \sum_{\beta=\alpha}^{16} u^{\alpha \beta}(t) P_{2}\left(Y_{\alpha}, Y_{\beta}\right)\right) \\
& +\sum_{\alpha=1}^{4}\left(u^{0 \alpha}(t)+\sum_{\beta=\alpha}^{16} u^{\alpha \beta}(t) P_{1}\left(Y_{\beta}\right)\right) P_{1}\left(X_{\alpha}\right) \\
& +\sum_{\alpha=1}^{4} \sum_{\alpha=i}^{4} u^{\alpha \beta} P_{2}\left(X_{\alpha}, X_{\beta}\right)
\end{aligned}
$$

where $P_{1}(x)=(2 x-1) / \sqrt{3}$ is the one-dimensional first order Legendre polynomial, while the second order two-dimensional modified Legendre polynomial is given by $P_{2}(x, y)=P_{1}(x) P_{1}(y)$ if $x \neq y$ and $P_{2}(x, x)=\left(6 x^{2}-6 x+1\right) / \sqrt{5}$. We rewrite the above expression as

$$
u(\boldsymbol{X}, \boldsymbol{Y}, t)=A^{0}(\boldsymbol{Y}, t)+\sum_{\alpha=1}^{4} A^{\alpha}(\boldsymbol{Y}, t) P_{1}\left(X_{\alpha}\right)+\sum_{\alpha=1}^{4} \sum_{\beta=1}^{4} A^{\alpha \beta}(t) P_{2}\left(X_{\alpha}, X_{\beta}\right)
$$

In the present example, we started by assuming that a full polynomial chaos decomposition is available, which we subsequently transformed into a chaos decomposition with random coefficients. In a context where experimental data is available, the random coefficients are directly estimated from measurements once the coarse variables $\boldsymbol{X}$ have been identified, without the need to go through the full polynomial chaos expansion (which would typically not be available). A variety of methods, such as Maximum Likelihood, Maximum 

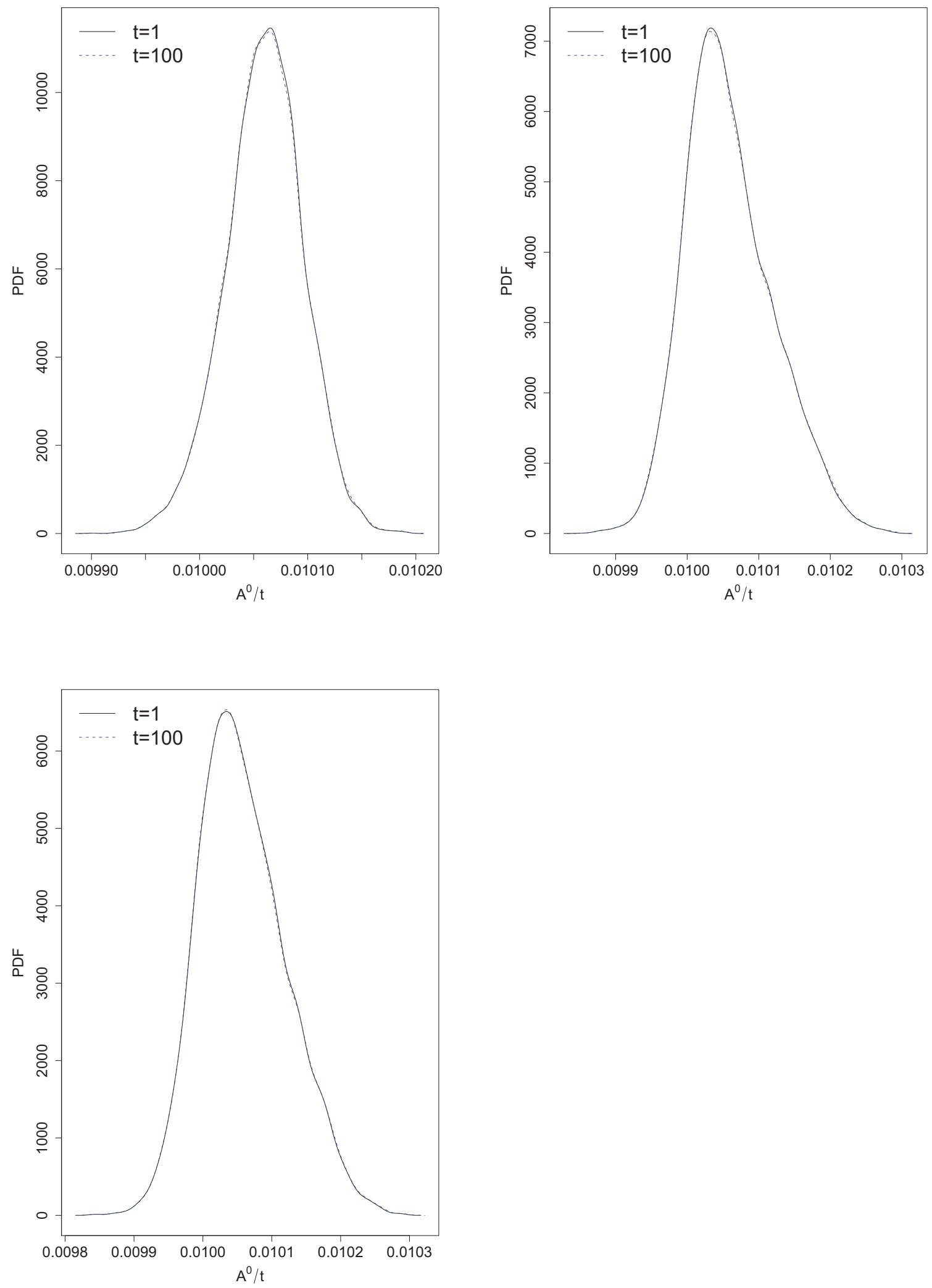

Fig. 1. Probability density function of the coefficient $A^{0}$ evaluated at two values of the index $t$; Three different sets of coarse scale variables; Top left: $\boldsymbol{X}=\left(\xi_{1}, \eta_{1}, \eta_{2}, \eta_{3}\right)$; Top right: $\boldsymbol{X}=\left(\xi_{2}, \eta_{23} \eta_{3}, \eta_{4}\right)$; Bottom left: $\boldsymbol{X}=\left(\xi_{4}, \eta_{8}, \eta_{9}, \eta_{10}\right)$. 
Entropy, or Bayesian inference $[6,10,4]$ can be used to estimate the sampling distributions of these reduced polynomial chaos coefficients. Figures (1)-(4) show probability distribution functions (PDF) for the coefficients $A^{\alpha}$ in equation (43), evaluated for at two end-points of the interval $\mathcal{T}$. Specifically, Figure (1) shows the term $A^{0}$ for each of the three cases described above. We observe that,as expected, the probabilistic scatter, as measured by the width of the PDF, increases as the quality of the coarse scale observables degrades. We also note that by showing the PDF of the quantity $A^{\alpha} / t$ (instead of just $A^{\alpha}$ ), the curves coalesce onto a single curve. The reason for this scaling has not been investigated and certainly merits further consideration. Figure (2) depicts PDF plots for $\left(A^{\alpha}, \alpha=1,2,3,4\right)$. Figures (3) and (4) show similar results for Cases 2 and 3, respectively. Except for $\alpha=1$ in Case 1, the same scaling behavior as described in previously is again observed. The observation made for Figure (1) in connection with uncertainty being inversely proportional to the quality of coarse observables (elements of vector $\boldsymbol{X}$ ) is reiterated for Figures (2)-(4).

\section{Conclusion}

We have developed a model reduction procedure for stochastic variables and processes. The procedure implements a model reduction by rolling insignificant dimensions into a distributional representation that does not allow their disentanglement, while permitting their separation from the other variables. Two procedures were theoretically described involving Karhunen-Loeve decompositions and Polynomial Chaos decompositions with stochastic coordinates. The proposed procedure has significant potential for representing and characterizing model error whereby the strength of the uncertainty in the estimated coefficients can be used as an indicator for the strength of the modeling error. We finally note that the foregoing development hinged on the linear form afforded for the solution when using polynomial chaos representations. 

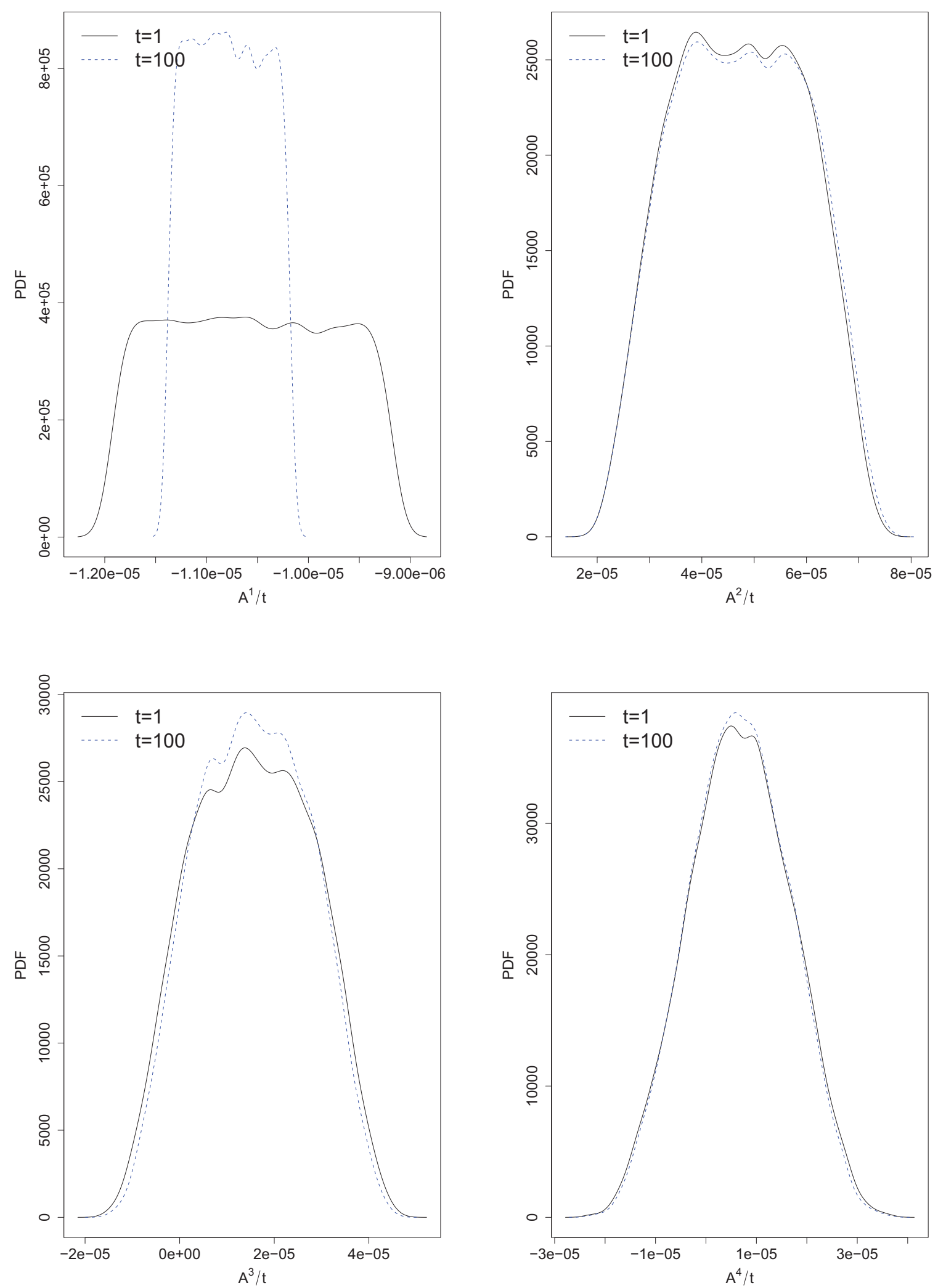

Fig. 2. Probability density function of the coefficient $A^{\alpha}$ evaluated at two values of the index $t ; \boldsymbol{X}=\left(\xi_{1}, \eta_{1}, \eta_{2}, \eta_{3}\right)$; Coefficients are shown for each of the four coarse scale variables. 

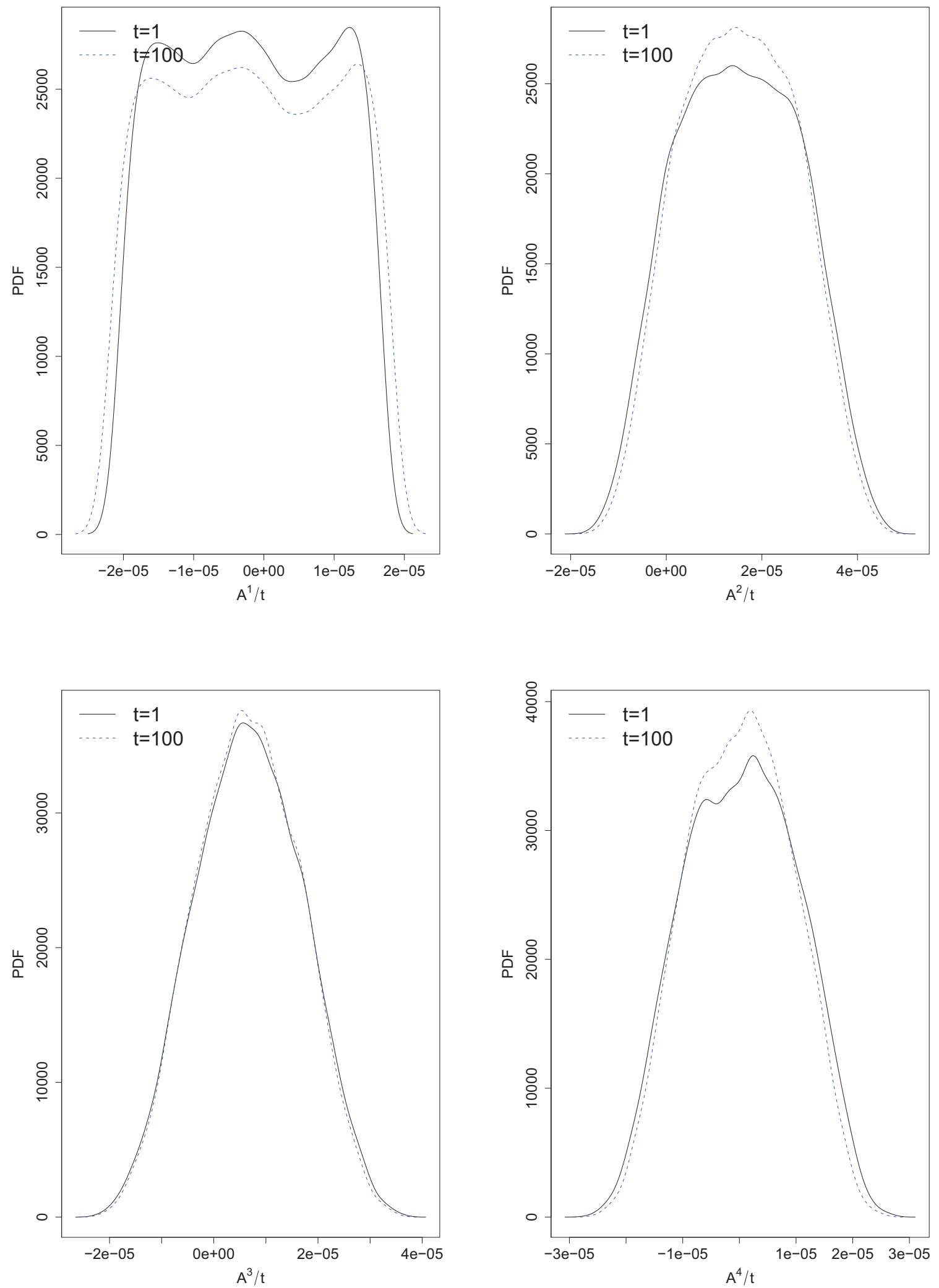

Fig. 3. Probability density function of the coefficient $A^{\alpha}$ evaluated at two values of the index $t ; \boldsymbol{X}=\left(\xi_{2}, \eta_{2}, \eta_{3}, \eta_{4}\right)$; Coefficients are shown for each of the four coarse scale variables. 

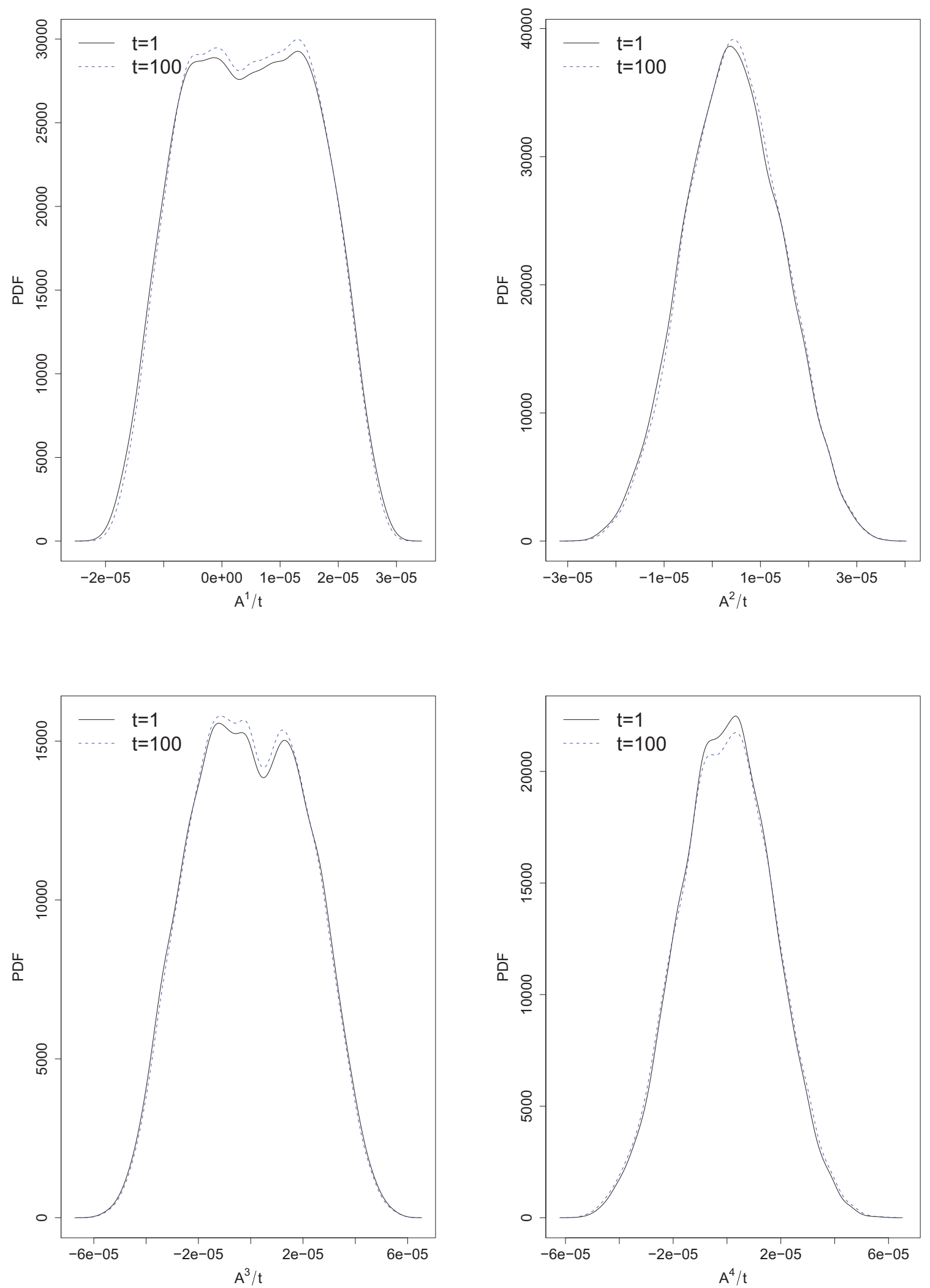

Fig. 4. Probability density function of the coefficient $A^{\alpha}$ evaluated at two values of the index $t ; \boldsymbol{X}=\left(\xi_{4}, \eta_{8}, \eta_{9}, \eta_{10}\right)$; Coefficients are shown for each of the four coarse scale variables. 


\section{References}

[1] M. Aarnst and R. Ghanem. Probabilistic equivalence and stochastic model reduction in multiscale analysis. Computer Methods in Applied Mechanics and Engineering, doi:10.1016/j.cma.2008.03.016, 2008.

[2] Velamur Asokan Badri Narayanan and Nicholas Zabaras. Variational multiscale stabilized FEM formulations for transport equations: stochastic advectiondiffusion and incompressible stochastic Navier-Stokes equations. J. Comput. Phys., 202(1):94-133, 2005.

[3] I. Babuska, R. Tempone, and GE Zouraris. Galerkin finite element approximations of stochastic elliptic partial differential equations. SIAM Journal on Numerical Analysis, 42(2):800-825, 2004.

[4] S. Das, R. Ghanem, and J. Spall. Asymptotic sampling distribution for polynomial chaos representation of data : A maximum-entropy and fisher information approach. SIAM Journal on Scientific Computing, 30(5):22072234, 2008.

[5] Sonjoy Das and Roger Ghanem. Hybrid representations for complex dynamical stochastic systems: coupled non-parametric and parametric models. In C. Soize and G.I. Schuller, editors, Proceedings of the $6^{\text {th }}$ international Conference on Structural Dynamics, pages v.1, pp.53-60, Paris, France, 4-7 September 2005.

[6] C. Descelliers, C. Soize, and R. Ghanem. Maximum likelihood estimation of stochastic chaos representation from experimental data. International Journal for Numerical Methods in Engineering, 66(6):978-1001, 2006.

[7] Philipp Frauenfelder, Christoph Schwab, and Radu Alexandru Todor. Finite elements for elliptic problems with stochastic coefficients. Computer Methods in Applied Mechanics and Engineering, 194(2-5):205-228, 2005.

[8] R. Ghanem. Ingredients for a general purpose stochastic finite elements formulation. Computer Methods in Applied Mechanics and Engineering, 168(14):19-34, 1999.

[9] R. Ghanem, A. Doostan, and J. Red-Horse. A probabilistic construction of model validation. Computer Methods in Applied Mechanics and Engineering, page doi:10.1016/j.cma.2007.08.029, 2008.

[10] R. Ghanem and R. Doostan. Characterization of stochastic system parameters from experimental data: A bayesian inference approach. Journal of Computational Physics, 217(1):63-81, 2006.

[11] R. Ghanem and P. Spanos. Stochastic Finite Elements: A Spectral Approach. Springer Verlag, 1991.

[12] M. Huebner and B. Rozovskii. On asymptotic properties of maximum likelihood estimators for parabolic stochastic pdes. Probability Theory and Related Fields, 103:143-163, 1995. 
[13] O.P. Le Maitre, H. Najm, R. Ghanem, and O. Knio. Multi-resolution analysis of Wiener-type uncertainty propagation schemes. J. Comp. Phys., 197(2):502-531, 2004.

[14] O.P. Le Maitre, Knio. O., H. Najm, and R. Ghanem. Uncertainty propagation using Wiener-Haar expansions. J. Comp. Phys., 197:28-57, 2004.

[15] H. G. Matthies and A. Keese. Galerkin methods for linear and nonlinear elliptic stochastic partial differential equations. Comput. Methods Appl. Mech. Engrg., 194(12-16):1295-1331, 2005.

[16] Habib N. Najm. Uncertainty quantification and polynomial chaos techniques in computational fluid dynamics. Annual Review of Fluid Mechanics, 41(1), 2009.

[17] J.R. Red-Horse and A.S. Benjamin. A probabilistic approach to uncertainty quantification with limited information. Reliability Engineering and System Safety, 85:183-190, 2004.

[18] E.V. Slud. The moment problem for polynomials forms in normal random variables. 21(4):2200-2214, October 1993.

[19] C. Soize. Random matrix theory and non-parametric model of random uncertainties in vibration analysis. J. Sound Vibration, 263(4):893-916, 2003.

[20] C. Soize. Random matrix theory for modeling uncertainties in computational mechanics. Comput. Methods Appl. Mech. Engrg., 194(12-16):1333-1366, 2005.

[21] C. Soize and R. Ghanem. Physical systems with random uncertainties: Chaos representations with arbitrary probability measure. SIAM Journal of Scientific Computing, 26(2):395-410, 2004.

[22] Xiaoliang Wan and George Em Karniadakis. Multi-element generalized polynomial chaos for arbitrary probability measures. SIAM J. Sci. Comput., 28(3):901-928 (electronic), 2006.

[23] D. Xiu and G. Karniadakis. Modeling uncertainty in flow simulations via generalized polynomial chaos. J. Comp. Phys., 187(1):137-167, 2003. 